\title{
STUDY ON PROCESSING PERFORMANCE OF A DPDK AND GPU COMBINED PULSAR DATA REDUCTION SYSTEM
}

\author{
Wei Dai ${ }^{1,3,4}{ }^{*}$, Feng Wang ${ }^{1,2,4}$ \\ ${ }^{1}$ Yunnan Astronomical Observatories, Chinese Academy of Sciences P.O.Box 110, Kunming 650011, China. \\ ${ }^{2}$ Center of Astrophysics, Guangzhou University, No.230, Waihuanxi Rd, Panyu District, Guangzhou 510006, China. \\ ${ }^{3}$ Key Lab of Computer Technology Application of Yunnan Province, Kunming University of Science and Technology, \\ No.727 South Jingming Rd., Chenggong District, Kunming 650500, China. \\ ${ }^{4}$ University of Chinese Academy of Sciences, 19A Yuquan Rd., Shijingshan District, Beijing 100049, China. \\ dweiwdai@yeah.net
}

\begin{abstract}
Pulsars are fast-rotating neutron stars which are some of the most extreme objects in the universe and important key probes for the fundamental physics. The massive amounts of data caused by high time resolution and wide bandwidth sampling poses a huge challenge to the realization of a reliable real-time process for pulsar observation systems. To achieve a real-time transmission and processing of up to several or even tens of gigabits per second for pulsar observations, this paper proposes a high performance and reliable real-time coherent dedispersion pipeline for the 40-m Radio Telescope of Yunnan Astronomical Observatories. We first introduce the specifications of the pulsar backend and analyze the requirements of coherent dedispersion pipeline of the telescope. After the design of the system architecture, we focus on the implementation of the high-performance pipeline. Two key techniques such as high-performance lossless data acquisition based on Data Plane Development Kit and real-time coherent dedispersion processing upon graphics processing unit platforms are presented in detail. The subsequent experiments and test observations demonstrate the proposed pipeline meets the requirements and enables long-term observations for the telescope.
\end{abstract}

Keywords: Methods: Data Analysis - Pulsars: General - Techniques: Miscellaneous.

\section{Introduction}

In recent years, the continuous emergence of new requirements for pulsar observation and research has resulted in a concurrent increase in requirements for real-time pulsar online reduction pipeline. On the one hand, the sampling accuracy and sampling bandwidths of analog-to-digital (ADC) boards are also constantly improving. On the other hand, the high-precision arrival times of pulsar signals and the microsecond- and nanosecond-scale structures of individual pulses demand wider bandwidths for improved sensitivity. For example, the current mainstream pulsar frontend has an observation bandwidth of 1-2 GHz, a sampling bit number of 8-12 bits, and an output data rate of up to several tens of $\mathrm{Gb} / \mathrm{s}$.

Receiving high volumes of data may consume significant compute resources and pose a huge challenge for data acquisition. It is therefore critical to minimize the resources required to receive streaming data. Reducing overhead allows more of resources to be dedicated to data processing. Traditional data acquisition programs generally use network system calls provided by the Linux operating system kernel to communicate with upstream systems. While modern Linux IP stack are well designed and implemented for security and robustness, user applications and system resources are strictly separated. Before data received from network can be accessed by user application, it will be copied several times and several interrupts and context switches will occur. In order to better cope with high-speed network environment, some network acceleration technologies, like Intel's Data Plane Development Kit (DPDK), net map, and PF RING, had been put forward. These frameworks allow line speed packet handling in user space on commodity hardware by kernel bypassing during packet processing while significantly decrease compute resources consumed. The higher performance makes these frameworks a promising basis for radio astronomical acquisition and processing software.

Apart from the high-rate receiving requirements for an online reduction pipeline, there are also high computational requirements need to be tackled. Astronomical data processing normally requires a large number of floating-point operations. At the beginning, dedicated hardware was built to perform real-time data processing. With the increasing performance of commodity computer hardware, 
software systems built with off-the-shelf hardware such as CPUs and GPUs have emerged. In the general information technology, data processing system constructed with MPI, Map Reduce, Spark and other frameworks have become ubiquitous. Using these existing solutions is a preferable scheme that would save time. However, they are not well suited to highrate and high-volume astronomical signal data stream processing. It is necessary to develop a framework to meet the data-handling requirements of future instruments and telescopes by combining high-rate data acquisition capability of network acceleration technologies with the high-performance data reduction capability of GPUs.

In this paper, we first review the technical features of some network acceleration technologies, such as Intel's Data Plane Development Kit (DPDK), net map, Packet map and PF RING. We then compare these frameworks inside a specific environment for radio astronomical data processing. A real-time pulsar data processing pipeline based on Intel's Data Plane Development Kit (DPDK) and Graphics Processing Units (GPUs) is discussed. The rest of the paper provides an implementation of the pulsar data processing pipeline for the YNAO-40M. Finally, Performance tests and test observations for system verification are presented. Whilst our work arises from the domain of pulsar astronomy, our processing scenario is closely related to real-time radio signal processing more generally. This work therefore has wide applicability.

\section{Related Work}

\subsection{Data acquisition system and user space I/O frameworks}

High-network IO introduces great challenges for data acquisition. Data acquisition systems that use traditional socket calls to receive data packets, such as PSRDADA (Kocz et al. 2015) and GUPPI DAQ DuPlain et al. (2008), experience frequent data loss and high system resource occupation under high traffic. Kernel parameters and net- work parameters must be adjusted to improve performance. Since the performance of the network protocol stack of mainstream operating systems has been unable to cope with the demand for $10 \mathrm{~Gb} / \mathrm{s}$ or higher network data transmission, some new network acceleration technologies have emerged that can allow network traffic to bypass the operating system kernel or reduce the number of copies in the memory. These techniques have been applied to several systems. For example, The Canadian Hydrogen Intensity Mapping Experiment (CHIME) telescope uses NTOPs proproletary PF RING/DNA technology (Recnik et al. 2015), and Versatile Green Bank Spectrometer (VEGAS) uses the packet mmap technology (David et al. 2018) to implement its HASHPIPE data capture package.

There were some features including zero-copy, kernel bypass, I/O batching, polling mode, exhibited by these user- space technologies. These features are key optimizations to achieve extreme performance. Zero-copy is a feature that aims to avoid memory-tomemory copy by mapping network interface card (NIC) memory to user space processes. Kernel bypass is bypassing the operation system's network stack and deliver raw packets directly into user applications. I/O batching is processing multiple packets with one API call on reception and sending.

Polling mode means accessing the RX and TX descriptors directly without any interrupts. Table 1 summarize the features of the $\mathrm{I} / 0$ frameworks we consider.

Table 1. Comparison of packet frameworks

\begin{tabular}{|c|c|c|c|c|}
\hline Framework & DPDK & Packet mmap & Netmap & PF RING ZC \\
\hline -Zero-copy & Y & $-\sim$ & Y & Y \\
\hline $\begin{array}{c}\text { Kernel } \\
\text { bypass }\end{array}$ & Y & N & Y & Y \\
\hline I/O Batching & Y & Y & Y & Y \\
\hline License & BSD & GPLv2 & BSD & Proprietary \\
\hline
\end{tabular}

A difference between these approaches is the feature set. Packet mmap is an extension added to the standard sockets in the Linux kernel, using a mmaped buffer, shared between user space and kernel. Packets are still processed by the whole kernel stack and could not keep in-kernel copy from happening. it does not have kernel bypass feature. Net map provides features including zero-copy, kernel bypass, batched I/O. However, the buer pool allocated to an application is not dynamic, which could prevent true zero-copy in some scenarios where the application must buer a lot of packets. PF RING ZC (ZeroCopy) is the combination of ntops PF RING and ntops LibZero. PF RING ZC supports huge pages and per-NUMA node buffer management. A major drawback is that PF RING ZC is not free and requires closed-source binaries.

The DPDK is an open-source technology introduced by Intel Corporation. It provides more user level functionalities such as a multi-core framework with enhanced NUMA-awareness, functions for packet manipulation across cores, mechanisms for memory anagement and interprocess communication etc. (Gallenmller et al. 2015). It also provides two execution model: a pipeline model and a run-to-completion model. DPDK can be considered more than just an I/O framework as it includes a packet scheduling and execution model that simplify application development. 
Many studies commonly concentrated on the comparison of frameworks' forwarding ability. Throughput measure- ments on commodity hardware conducted previously had shown that DPDK is the fastest in term of $\mathrm{I} / 0$ throughput. Furthermore, this paper concentrates on receiving, multithreading and multicore application under NUMA environ- ments, we decided to use DPDK as platform for our implementation.

\subsection{Dedispersion system and dedispersion techniques}

The coherent dedispersion of pulsar signals is one chief approach to the observation of pulsars.

As the electromagnetic waves radiated by pulsars propagate through the interstellar medium, different frequencies of the electromagnetic waves experience different delays, which broadens the observed pulsar profile.

Coherent dedispersion transforms the time domain signal into the frequency domain via Fourier transform, dedisperses it in the frequency domain, transforms it back to the time domain via inverse Fourier transform, and folds it according to the spin period. Coherent dedispersion can completely eliminate the in-band dispersion and obtain the original signals. Although coherent dedispersion is hindered by problems such as large data volumes, long processing periods, and limited bandwidths, it can preserve pulsar signal phase information, which is highly beneficial to the corresponding scientific research work.

A few dedispersion systems continue to be demonstrated around the world. The Green Bank Ultimate Pulsar Processing Instrument (GUPPI) in the United States can process an $800 \mathrm{MHz}$ bandwidth and 8-bit quantization (i.e., $1600 \mathrm{MB} / \mathrm{s}$ ) data in real time (DuPlain et al. 2008). Europe is developing a new generation of digital wideband coherent dedispersion systems based on the general highperformance computing platform UniBoard( Hargreaves 2012). The PuMa-II backend at the Westerbork Synthesis Radio Telescope in the Netherlands uses a CPU cluster to implement a near real-time coherent dedispersion system with a bandwidth of $160 \mathrm{MHz}$ (Karuppusamy et al. 2008).

The pulsar observation terminals currently used in China are the PDFB observation systems introduced by the National Astronomical Observatory and the Xinjiang Observatory from Australia, which support an incoherent dedispersion mode (Nie et al. 2017).

The Collaboration for Astronomical Signal and Electronics Research (CAPSER) of the University of Berkeley has released a series of Field Programmable Gate Array (FPGA) boards to the radio-astronomy community (Hickish et al. 2016).
The National Radio Astronomy Observatory designed and built a Digital Backend System based on CASPERs ROACH2 frontend and a heterogeneous computing backend (Ford et al. 2014), which supports incoherent pulsar search modes and coherent dedispersion timing modes.

Many pulsar observation technologies adopt high-speed ADC converters to realize wideband data acquisition. These systems achieve baseband conversion with a polyphase filter on the FPGA platform and realize data transmission with Ethernet technology, using general computer clusters to enable real-time data processing. As frontend signal acquisition capabilities continue to improve, multiple high-speed Ethernet interfaces have become commonly used to output data, which places enormous pressure on the backend data acquisition and processing systems.

For the fundamental research on observational mode, due to the influence of the interstellar medium, the pulsar radiation signals with different frequencies are dispersed during transmission. As a result, high-frequency signals reach the earth earlier than low-frequency signals. This broadens the pulse and lowers the signal-to-noise ratio (Lorimer \& Kramer 2005). The effect of dispersion on a radio signal can be described by a signal passing through a filter with an interstellar medium transfer function (Hankins \& Rickett 2012; Hankins 1971):

$$
H\left(v_{0}+v\right)=e^{i \frac{2 \pi v^{2} D M}{v_{0}^{2}\left(v_{0}+v\right)} k_{d m}}
$$

where $v_{0}$ is the center frequency of a limited bandwidth $\Delta \mathrm{v}, \mathrm{v}$ is the offset to $\Delta \mathrm{v}$, and $-\Delta \mathrm{v} / 2<$ $\mathrm{v}<\Delta \mathrm{v} / 2$. The observable dispersion measure DM is the integrated density of free electrons along the line of sight, which is usually expressed in $\mathrm{cm}^{-3} \mathrm{pc}$, and $\mathrm{k}^{-1} \mathrm{dm}=2.41 \times 10^{-4} \mathrm{sMHz} 2$ (Manchester \& Taylor 1972).

There are two main dedispersion techniques: incoherent and coherent dedispersion. Incoherent dedispersion splits the observable bandwidth into a number of frequency channels and align the pulses in these channels by adding appropriate time delays. It is clear that incoherent dedispersion does not remove the intra-channel dispersion delay.

To completely eliminate the dispersion effect, coherent dedispersion passes the observed signal through a filter with an inverse function of the interstellar medium transfer function.

Thus, coherent dedispersion can produce greater signal-to-noise sensitivity at the cost of higher processing requirements.

In a summary, there are quite a few literatures on pulsar observational system and coherent dedispersion data processing.

However, few have discussed high performance lossless data acquisition using DPDK which can greatly improve data acquisition performance and provide an efficient parallel framework. 


\section{System Architecture Design}

\subsection{Requirement Analysis}

We systematically investigated the requirements of YNAO-40M pulsar observation.

The main requirements are mainly focused on software because it is impossible to replace all original hardware due to the lack of construction funds available.

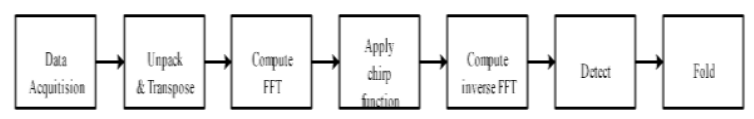

Figure 1: Coherent dedispersion data processing flowchart.

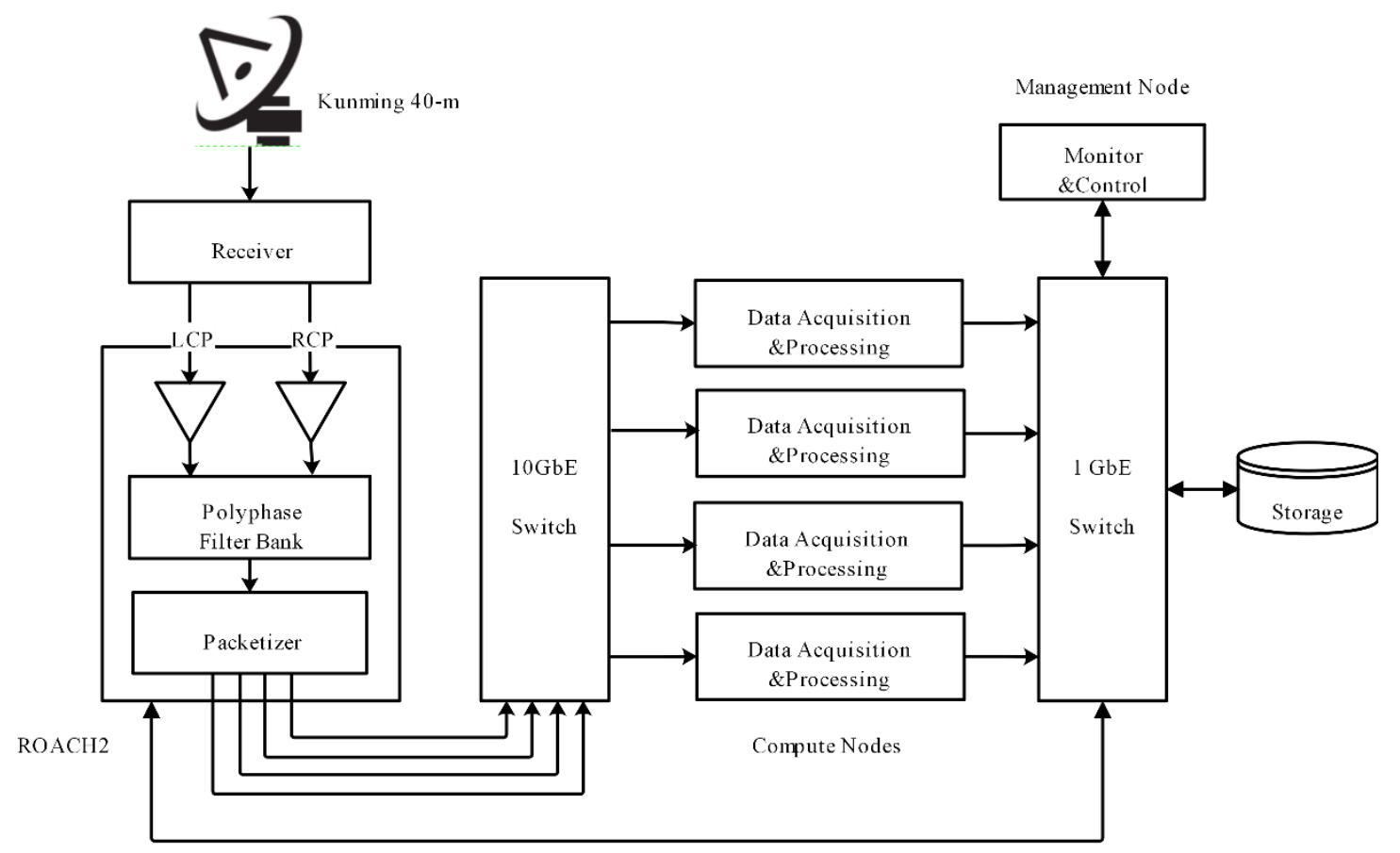

Figure 2: Diagram of the pulsar data processing system

A CASPER ROACH 2 board equipped with dual 5 Gsps ADCs performs the initial analog-to-digital conversion on Left Circular Polarization (LCP) and Right Circular Polarization (RCP) inputs. After applying channelization via polyphase filter bank, the output is a 128-channel digital baseband signal, and the bandwidth of each channel is $4 \mathrm{MHz}$. Each signal is requantized to 8 bits real and 8 bits imaginary. The 128-channel data is divided into four subbands and separately packaged. Header information is added to packaged data to facilitate data decoding. The packet streams are transmitted via UDP packets to four computer nodes for further processing, each receiving a different 32-channel subset of data (Lan et al. 2016).

The $\mathrm{ROACH}_{2}$ board and all servers are connected via a 10 Gigabit Ethernet $(10 \mathrm{GbE})$ network. Fullcrossbar connectivity is provided by an $\mathrm{H}_{3} \mathrm{C}$ S630042QT ethernet switch.

A high-performance cluster which consists of four servers was built for coherent dedispersion processing. Each server has a 2 -way $2.3 \mathrm{GHz}$ Intel Xeon E5-2698 v3 processor, 32 cores, 256 GB memory, and a 1 TB hard disk. Two NVIDIA GeForce GTX TITAN-X GPU cards are installed in each server respectively. Each machine also has a $10 \mathrm{GbE}$ interface and a $1 \mathrm{GbE}$ interface. The operating system CentOS 6.9 is installed on each node.

Each machine simultaneously receives one-fourth of these bandwidths as a $128 \mathrm{MHz}$ subband of complex voltage data on its $10 \mathrm{GbE}$ interface from the $\mathrm{ROACH}_{2}$ board as a stream of UDP packets. Coherent dedispersion modes uses the GPUs in the HPC machines to run a dedispersion filter for each channel in real time and to fold at the pulsar period.

The $\mathrm{ROACH}_{2}$ board and all servers are also attached to another Ethernet switch. A management node based on Python can send commands to control the $\mathrm{ROACH}_{2}$ boards and HPC nodes. Userspecified inputs and configuration information kept in configuration files are used to setup and initialize the pipeline. Folded profiles from the HPC nodes are collected and written to a disk. The results are written in the PSRFITS format (Hotan et al. 2004).

\section{Design and Implementation}

The coherent dedispersion process involves a large number of floating-point operations. Thus, parallel processing is the only feasible method to perform data processing in real time. The parallel method proposed in this paper is performed at three levels. 
The first level of parallelism is cluster parallelism, where the FPGA platform splits the baseband data into different subbands and distributes them to cluster nodes. The nodes synchronize their data processing via timestamps marked in the frame header.

The second level of parallelism is thread parallelism, where a number of threads belonging to a single process communicate collectively via shared memory on a subband data stream.
The third level is the exploitation of the parallel processing power of the GPUs. In our implementation, a copy of the pipeline, which is mainly written in $\mathrm{C}$ and $\mathrm{C}++$, is launched on each computer server.

The processing pipeline is divided into four different stages. As show in Figure 3 , blocks of data are processed and then transferred into the pipeline via shared memory. The details of the individual stages are as follows:

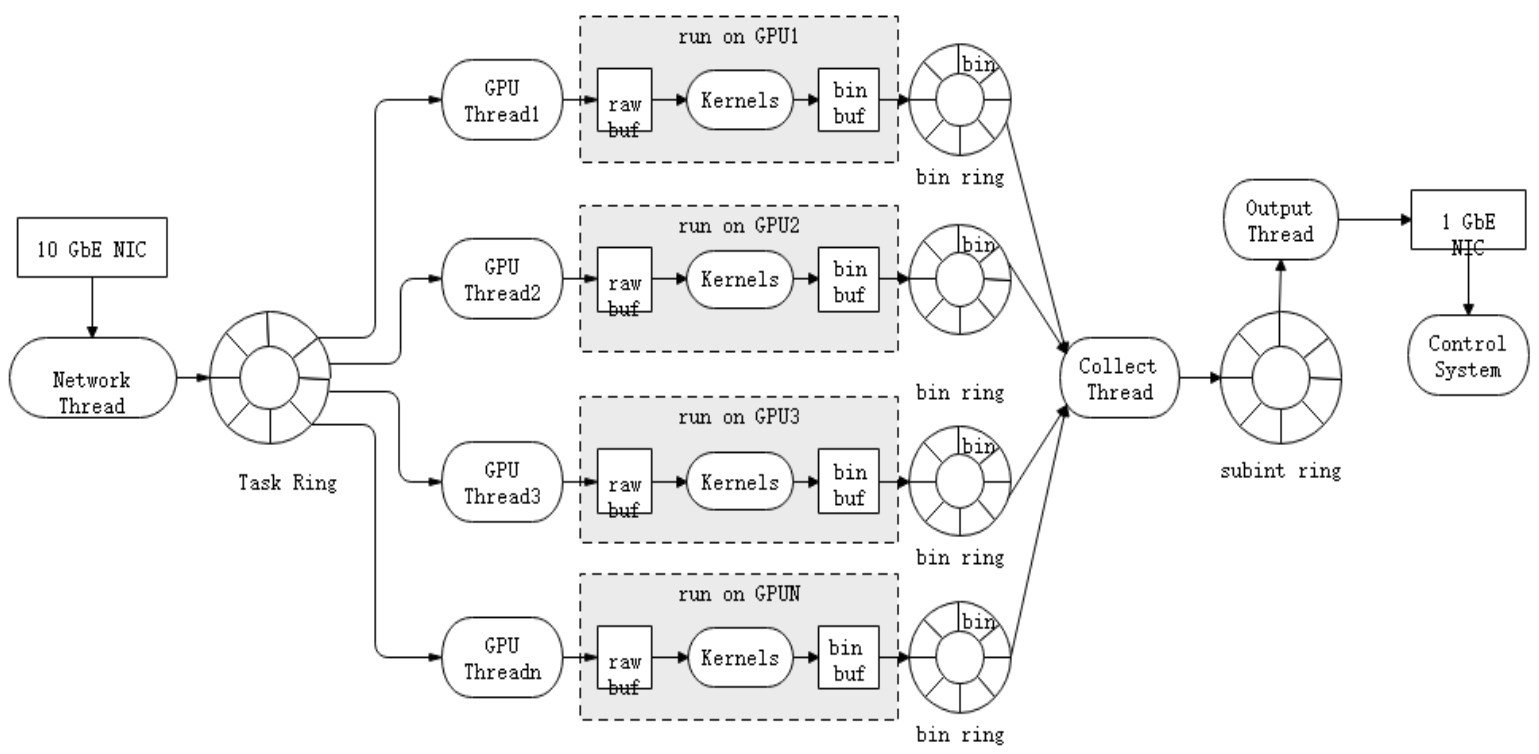

Figure 3: Parallel processing of de-dispersion on one compute node

Data capture and buffer: The starting point of the local pipeline is the capture of a time series of a subband encapsulated in the UDP packet stream.

The pipeline is responsible for capturing UDP packets and parsing their headers to determine how to divide the continuous packet contents into blocks of data.

The packets all have timestamps and sequence numbers such that they can be reordered into the proper time sequence if they arrive out of order from the network.

To balance the computational load, these blocks of data are written into a lockless single-producer and multi-consumer ring buffer in the shared memory. The ring buffer allows multiple threads to each remove data and allows the network threads to write data simultaneously.

Coherent dedispersion and fold: In this stage, the chirp function is initialized and the time series is read from the ring buffer.

The number of dedispersion threads is determined by the number of GPUs, and each thread is responsible for managing one GPU card. These threads fetch tasks from the ring buffer independently. Every thread takes one task at a time, then copies the task to the GPU and performs dedispersion and folding.
The average profile is copied back to the host memory and placed into a separate ring buffer.

Collect and merge: In the previous phase, data blocks are processed by multiple threads from the queue according to the First In First Out (FIFO) rule.

The collection thread takes the results stored in the buffers of the respective threads in chronological order and merges them into sub-integrations.

Send to management node: Sub-integrations produced by different nodes must be combined and written to a file on the management node.

An output thread acquires the sub-integrations from the ring buffer and sends them to management node through a TCP connection via another network adapter.

\subsection{Data capture and buffering}

To acquire the massive data produced by $\mathrm{ROACH}_{2}$, the server receives the observational data encapsulated as a frame through a $10 \mathrm{~Gb}$ optical fiber.

The frame includes 32 channels of observational data with 2 polarizations.

The data arrangement of the data frame format is shown in Figure 4. 


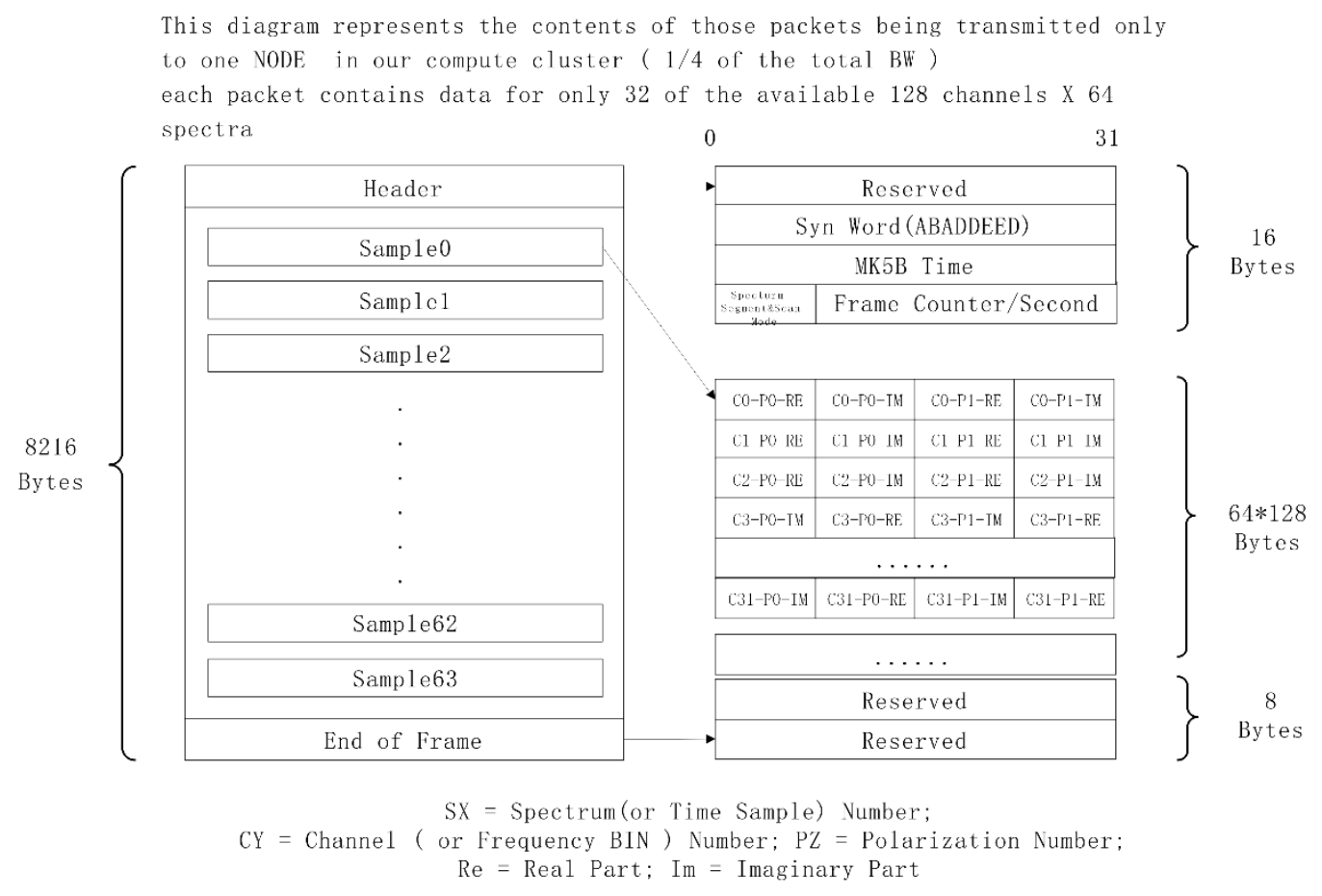

Figure 4: Data frame format

SX represents the spectrum number, each data frame in the design encapsulates 64 spectra; CY is the channel number, each data frame in the design packages 32 channels; PZ is the polarization number, either left-handed or right-handed; and Re is the real part of the sampling point data and Im is the imaginary part. The design has 128 channels, with each 32-channel data using an independent $10 \mathrm{~Gb}$ network transmission. The bandwidth of the data transmitted by each $10 \mathrm{G}$ network interface is onefourth of the total bandwidth. In radio astronomy, the digital baseband conversion terminal has strict requirements for the signal reception time, which must be encapsulated in the data frame. Therefore, in the $\mathrm{ROACH}_{2}$ FPGA design, the time formatter employs a modified Julian day format. The ROACH2 time formatter must synchronize with the GPS time. To allow for the transmission of a packetized stream of UDP packets with data payloads as large as 8216 bytes, jumbo frames are used by setting the MTU to 9000 bytes. Jumbo frames are Ethernet frames with more than 1,500 bytes of payload.

Each computer node must capture UDP packets with minimal overhead and without loss. It is therefore necessary to investigate methods to minimize the resources required for the capture of streaming data. Reducing the data transfer overhead allows for more of the resources to be dedicated to data processing.

We designed and implemented a data acquisition module based on DPDK which is shown in Figure 5. One network thread runs on a dedicated core. The network thread acquires network packets through polling mode instead of interrupt mode. In interrupt mode, the network adapter generates an interrupt every time it receives a frame to notify the CPU to process. According to the calculation of 1500 bytes per Ethernet frame, the number of interruptions per second is about 6.6 million at $10 \mathrm{~Gb} / \mathrm{s}$. On the other hand, polling mode shields the hardware interrupt and directly queries the network card to obtain data packets, which avoids any unnecessary interrupt processing overhead and eliminates kernel from the data transfer path.SX represents the spectrum number, each data frame in the design encapsulates 64 spectra; CY is the channel number, each data frame in the design packages 32 channels; PZ is the polarization number, either left-handed or righthanded; and Re is the real part of the sampling point data and Im is the imaginary part. The design has 128 channels, with each 32-channel data using an independent $10 \mathrm{~Gb}$ network transmission. The bandwidth of the data transmitted by each $10 \mathrm{G}$ network interface is one-fourth of the total bandwidth. In radio astronomy, the digital baseband conversion terminal has strict requirements for the signal reception time, which must be encapsulated in the data frame. Therefore, in the $\mathrm{ROACH}_{2}$ FPGA design, the time formatter employs a modified Julian day format. The $\mathrm{ROACH}_{2}$ time formatter must synchronize with the GPS time. To allow for the transmission of a packetized stream of UDP packets with data payloads as large as 8216 bytes, jumbo frames are used by setting the MTU to 9000 bytes. Jumbo frames are Ethernet frames with more than 1,500 bytes of payload.

Each computer node must capture UDP packets with minimal overhead and without loss. It is 
therefore necessary to investigate methods to minimize the resources required for the capture of streaming data. Reducing the data transfer overhead allows for more of the resources to be dedicated to data processing.

We designed and implemented a data acquisition module based on DPDK which is shown in Fig. 5. One network thread runs on a dedicated core. The network thread acquires network packets through polling mode instead of interrupt mode. In interrupt mode, the network adapter generates an interrupt every time it receives a frame to notify the CPU to process. According to the calculation of 1500 bytes per Ethernet frame, the number of interruptions per second is about 6.6 million at $10 \mathrm{~Gb} / \mathrm{s}$. On the other hand, polling mode shields the hardware interrupt and directly queries the network card to obtain data packets, which avoids any unnecessary interrupt processing overhead and eliminates kernel from the data transfer path.

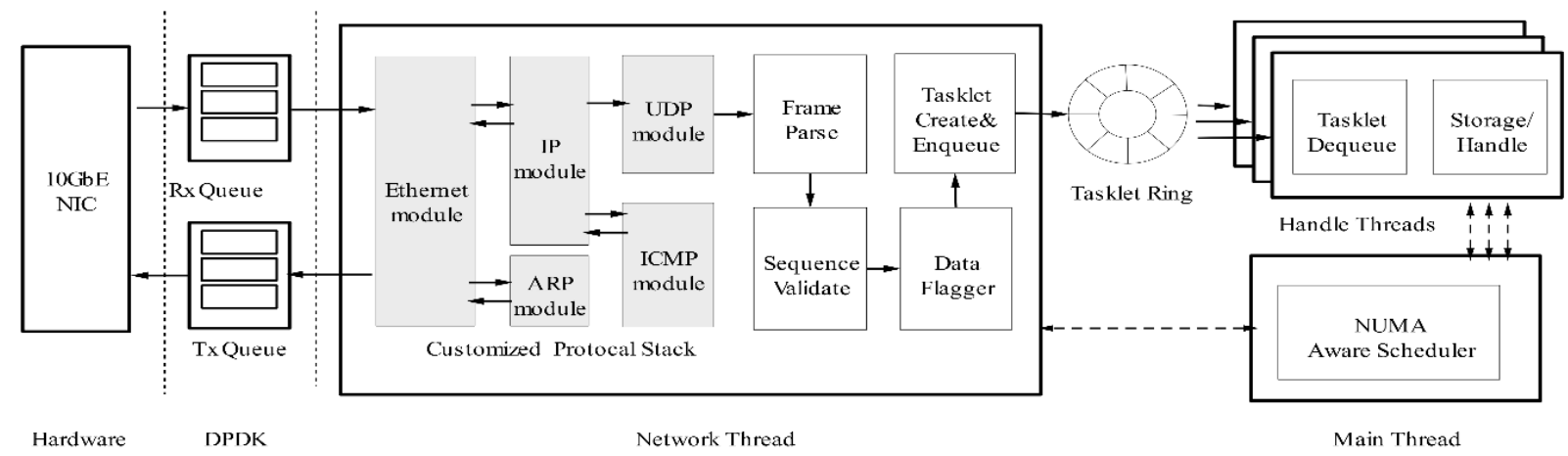

Figure 5: Diagram of high-performance data acquisition for pulsar observation

When using the DPDK polling-mode driver to receive packets, all packets pass directly into the user space application and do not traverse the kernel IP stack. The application was written to understand TCP/IP protocols. Since our architecture emphasizes the unidirectional high-throughput transfer of UDP packets, an application-tailored network stack was implemented to support minimal features including ARP, IP, ICMP, and UDP protocols. Therefore, when the network thread is initialized, network parameters such as MTU, IP address, network mask, and default gateway are set according to the configuration information, and the operating system settings are not used. After the packets are decapsulated by Ethernet, IP, and UDP, they are checked and parsed according to the format shown in Figure 5. The network thread then performs packet loss detection and rearrangement based on the timestamp and the in-second count and places the UDP payload into a tasklet. The tasklet is a custom data block object that can be processed independently. In addition to the data, it also describes the metainformation related to data processing such as the start timestamp, sample size, center frequency, and dispersion measurement. Once a tasklet is full, it is placed into a queue, and an empty tasklet is retrieved from the tasklet pool.

Since data reception, parsing, and distribution involve a large number of memory operations, data objects such as tasklets are created and managed by object pools. These pools initialize and manage objects in the huge page memory instead of standard page memory. As a result, the performance is increased since pre-allocated objects can be easily reused and translations from virtual to physical page addresses are significantly reduced.

Modern computers usually have a non-uniform memory access (NUMA) architecture, in which memory regions and PCle devices are more closely associated with a specific CPU socket than with others. To achieve the best performance, the framework pins the network thread on the CPU core that handles the $10 \mathrm{G}$ network adapter interrupts and constructs object pools in the memory regions associated with the same CPU core.

\subsection{Parallelizing Coherent Dedispersion on Multi-GPU Devices}

The baseband data is divided into $n_{\text {subband }}$ dualpolarized Nyquist complex-sampled frequency subbands, each of which consists of $n_{\text {subchannel }}$ channels. Each computer node is responsible for receiving and processing the UDP data stream corresponding to one subband. The algorithm uses multiple GPU devices for unpacking, Fourier transforms, dispersion, inverse Fourier transforms, detection, and folding. The details of the algorithm are described as follows:

1. Initialize the running parameters. Calculate the chirp function according to dedispersion measure value. Calculate the decoding table.

2. Create GPU threads according to the number of available GPUs. Each GPU thread uses one GPU device, and the following routine is executed by multiple threads in parallel:

(a) Copy decoding table and chirp function from host memory to GPU device memory. 
(b) Acquire a task from the task queue. The length of data contained in the task is $n, n=n_{\text {channel }} \times$ $n_{\text {pol }} \times n_{\text {dat }} \times n_{\text {dim }}$, where $n_{\text {channel }}$ is the number of channels, $n_{\text {pol }}$ is the number of pols, and $n_{\text {dat }}$ is the number of samples. Currently, $n_{\operatorname{dim}}=2$, which indicates complex sampling. $n_{\text {dat }}=\left(n_{f f t l e n}-n_{D M}\right) \times$ $n_{\text {batch }}+n_{D M}$.

(c) If the timestamp of the task is less than the observation start time, discard the task and return to step (b).

(d) If the timestamp of the task is greater than the observation end time, discard the task and proceed to step (o).

(e) Copy the task to the GPU device and execute steps (f) through (m) on the GPU.

(f) Decode the data and transpose the data from time, frequency, and polarization arrangement to frequency, polarization, and time arrangement.

(g) Perform Fourier transforms on each channel for each polarization to the frequency domain. Split data into $n_{\text {batch }}$ chunks of length $n_{\text {fftlen }}$ and execute multiple Fourier transforms simultaneously in batch mode. The length of the overlap region between adjacent chunks is $n_{D \mathrm{M}}$.

(h) Multiply frequency domain values with the chirp function to remove the dispersion.

(i) Apply inverse Fourier transforms to the data to return the signal to the time domain.

(j) For the results of all inverse Fourier transforms in step (j), discard $n_{D M} / 2$ points from the head and tail and output the remaining $n_{f f t l e n}-$ $n_{D M}$ values.

(k) Compute the polarization.

(l) Divide the pulse period equally into nbin bins. Use the TEMPO2 package to generate the pulsar timing model (Edwards et al. 2006). Calculate the phase of every sample relative to the pulse period and add the sample to the corresponding bin.

(m) Transfer the folding result to host memory.

(n) Place the folding result into the ring buffer and return to step (b).

(o) Thread exits.

Multiplying the Fourier transform of the baseband data with the chirp function in the frequency domain is equivalent to convolving the baseband data with the time domain form of the chirp function. Thus, the length of the Fourier transform sampled data is selected as follows. If the bandwidth of the signal for coherent dedispersion processing is $\Delta f$, the number of data points ndm required for coherent dedispersion is related to the dispersion delay. $n_{D M}=t_{D M} \Delta f$, and $t_{D M} \sim=8.3 \times$ $10^{6} \mathrm{~ms} \times D M \times \Delta f \times f^{3}$. For the discrete convolution process, if the length of the time series is $n$, then every point in the convolution is related to $n / 2$ points in the sequence. Therefore, it is necessary to use at least $2 n_{D M}$ data points for the coherent dispersion calculation. To improve the efficiency of the calculation, nfftlen is greater than $2 n_{D M}$ in steps (b) and (h).
Further, the time domain data obtained in step (i) is equivalent to the circular convolution in the time domain. The linear convolution result is required here, so the data after $n_{D M}$ point is removed in step (j) to afford the valid data. $n_{D M}$ is the number of points corresponding to the dispersion delay in the channel. To obtain continuous time-domain data, the overlap-save method is usually used to overlap each chunk with the front and back chunks with an overlap of $n_{D M}$ points (Bassa et al. 2017; Van Straten $\&$ Bailes 2011). In this paper, cuFFTs advanced data layout is used to perform Fourier transforms in batch mode with overlap inputs. The overlap between tasks is pre-copied and filled by network threads.

Network transmission, memory copy, and floating point calculations use a large amount of system resources. When implementing the algorithm, the following tuning methods are adopted to reduce the system overhead.

Thread parallel optimization based on NUMA topology: When the GPU processing thread is created, the master thread preferentially allocates the CPU core on the NUMA node where the GPU is located, reducing the overhead caused by thread switching and memory transmission across the NUMA node.

Ring buffer and memory pool management based on huge page memory: The threads in the pipeline communicate through a ring buffer. We use lockless circular queue of DPDK to implement the ring buffer. The task ring buffer is a single producer multiconsumer queue, and the bin ring buffer and the subintegration ring buffer are queues for singleproducer single-consumption. Both the ring buffer and the memory pool use large contiguous physical memory allocated via the hugetlbfs kernel file system.

GPU memory transfer and asynchronous execution optimization: Since there is a large amount of data exchange between the CPU and the GPU, memory transfer can easily become a bottleneck. The unpack and fold steps are implemented on the GPU, which can effectively reduce the amount of data transmitted. We also optimize the GPU code with concurrent memory transfers and kernel executions by using streams. Taking 32-channel dual-polarized data as an example, the length of the unpacked results from $250 \mathrm{MB}$ data is $1 \mathrm{~GB}$, and the final size is $524 \mathrm{~KB}$ after folding by 1024 bins.

\section{Performance and Test Observation}

To verify the performance of the pipeline, the data transfer and real-time processing performances were first tested. We then performed test observation on the telescope to verify the overall availability of the pipeline. 


\subsection{Data acquisition}

We first compared the data transfer performance of both the DPDK and UDP implementations. Our test platform comprised a data-sending server and a data-receiving server connected with a H3C S630042QT Ethernet switch. Both servers were equipped with Intel E5-2630 v3 CPUs running at $2.4 \mathrm{GHz}$, with 256 GB RAM and an Intel 82599ES Ethernet card.

We chose jumbo frame to transmit UDP packets with lengths of $9 \mathrm{~K}$ bytes for testing so as to guarantee the efficiency of transmission. The packet loss rates at different data rates from $1 \mathrm{~Gb} / \mathrm{s}$ to 10 $\mathrm{Gb} / \mathrm{s}$ were tested separately with a test duration of 1 minute.

\begin{tabular}{|c|c|c|c|c|c|c|c|c|c|c|}
\hline Throughput Gb/s & 1 & 2 & 3 & 4 & 5 & 6 & 7 & 8 & 9 & 10 \\
\hline $\begin{array}{c}\text { Loss rate of Socket- } \\
\text { based acquisition }\end{array}$ & 0 & 0 & 0.04 & 0.2 & 0.3 & 0.4 & 0.5 & 0.8 & 0.9 & 1.2 \\
\hline $\begin{array}{c}\text { Loss rate of DPDK- } \\
\text { based acquisition }\end{array}$ & 0 & 0 & 0 & 0 & 0 & 0 & 0 & 0 & 0 & 0 \\
\hline
\end{tabular}

\subsection{Coherent dedispersion processing}

A test server was configured with NVIDIAs K80 graphics processor unit. The K80 graphics processor consists of two GK210s with a total of 24 GB memory and 4,992 CUDA cores.

We selected off-line data of six pulsars with different dedispersion measuret (DM) values for testing.
The experimental results are shown in Table 2.

The tests showed that the DPDK implementation can capture packets without packet loss at speeds of up to $10 \mathrm{~Gb} / \mathrm{s}$, which clearly exceeds the requisite 4 $\mathrm{Gb} / \mathrm{s}$. The sequence numbers were tracked to ensure that lost packets could be detected. In addition, we also tested the stability of the DPDK-based implementation at a data rate of $10 \mathrm{~Gb} / \mathrm{s}$.

In a test with a duration of 2 hours, the number of lost packets was 0 , which indicated that the DPDKbased implementation has a very good stability and can meet the high IO data transmission requirements in a pulsar observation system.

Table 2. Comparison of packet loss rate /\% 


\subsection{Test observation}

This test was conducted with the YNAO-40M radio telescope. Two compute nodes are used to process $256 \mathrm{MHz}$ bandwidth.

Observations of three pulsars were carried out: J1136+1551 with an observation time of $30 \mathrm{~min}$ and J1932+1059 and J2022+5154 with observation times of $15 \mathrm{~min}$. The sub integration time was $10 \mathrm{~s}$.

The pipeline on every nodes used two GPUs for

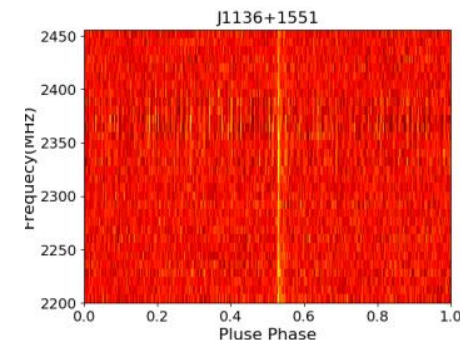

(a)

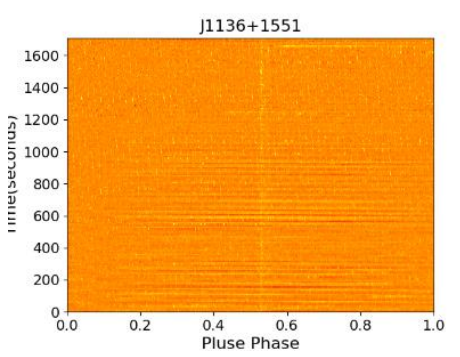

(d)

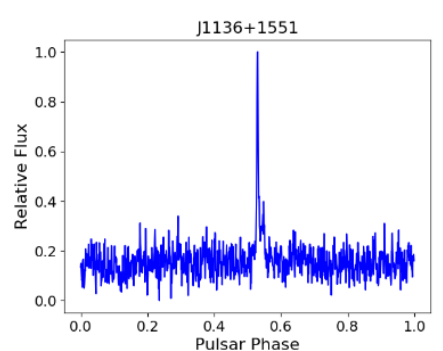

(g)

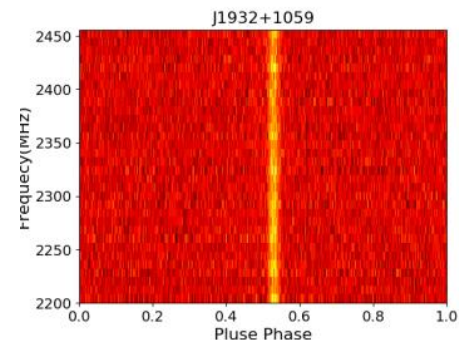

(b)

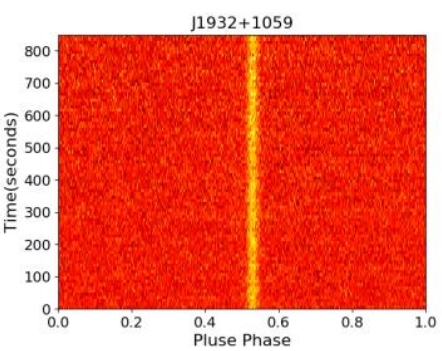

(e)

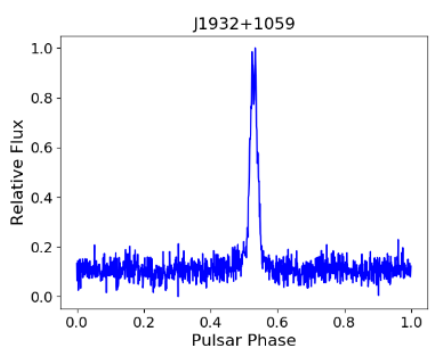

(h) processing, called TEMPO2 for periodic prediction, folded into 1024 bins, and finally sent the data to the management node for output as PSRFITS format files. The output file sizes were $354 \mathrm{MB}$ for $\mathrm{J} 1136+1551$ and $196 \mathrm{MB}$ for J1932+1059 and J2022+5154. The 8-bit complex voltages were processed into reduced data products using our realtime pipeline, thus reducing the data to approximately $0.04 \%$ of its original size.

The pulse profile diagrams are shown in Figure 6.

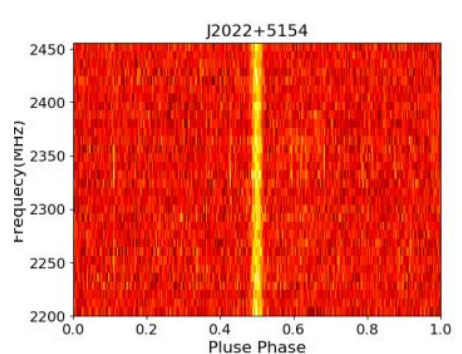

(c)

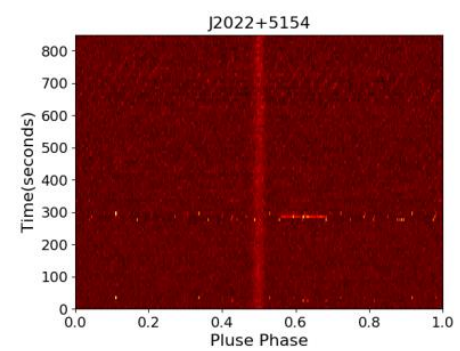

(f)

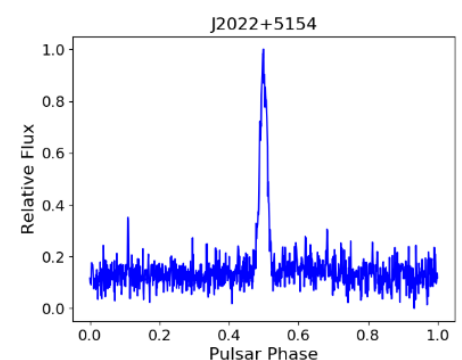

(i)

Figure 6: Observation results of J1136+1551, J1932+1059, and J2022+5154. (a)(b)(c)Frequency-phase plots;

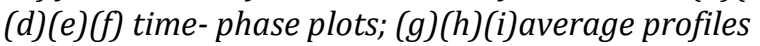

During the observation, our pipeline performed coherent dedispersion at better than real-time data acquisition rates with the current bandwidths.

This real-time pipeline also performed reliably without losing any data. No packet loss was observed by checking the timestamps and sequence numbers of the data frames. After the data acquisition and real- time processing by our pipeline, we obtained the pulse profile diagrams of the three radio source.

In the pulse profile diagrams, the double-peak structure of J1136+1551 and J1932+1059 and the unique structure of $\mathrm{J} 2022+5154$ are clearly observable.
A comparison of the average profiles to those released by the European Pulsar Network confirmed that the observation was successful.

\section{Discussion}

From the experimental and experimental observations, the development of the entire system was successful.

However, some feature should be discussed in detail, some of them could be further optimized in the near future. 


\section{Scalability}

Current design and implementation of the pipeline has very good scalability. On the one hand, the computing node can be added or expanded according to the amount of the bandwidth needs to be processed. It is very easy to construct a system with load balance and high compatibility. On the other hand, the system is a loosed couple system. All algorithms have been implemented by relatively independent processing component which could expand processing function easily and quickly.

\section{Data Consistency}

For a distributed data processing system, how to guarantee the data consistency is a critical problem. The consistency means that the processing results for the data at a certain observational time, no matter how many GPUs processed, should be ensured consistent and correct. Even if one node has the delay for computing, the final result should be kept correct as well.

Refer to other data processing system, we set a timestamp in the data received from $\mathrm{ROACH} 2$. The timestamp information would be wrapped throughout the entire data processing process. The results are stored in the proper file and position according to the timestamp information.

\section{DPDK vs other techniques}

The problems of data transmission are seldom discussed in previous literature. For many regular pulsar observational systems, UDP datagram with large size is an effective solution for data transmission. However, many potential problems would be appeared when we meet massive amount of the data. The CPU usage and memory usage would significantly increase while processing. At some point, the system will appear very short pause because of the PCI bus interruption. DPDK is actually an effective technique which could decrease the system consumption and guarantee the transmission performance. Frankly speaking, the software development using DPDK is indeed a challenge task. But experimental results demonstrated that the investment in the DPDK is very worthwhile. Actually, for a specified data collection system, it is not necessary to implement a comprehensive network stack. A minimal UDP stack is enough for the highperformance data acquisition for pulsar data collection. Accelerated with kernel by pass network drivers of DPDK, a lockless ring buffer, and memory pools, our pipeline can easily receive and buffer lossless high-volume data at line rate on $10 \mathrm{GbE}$ links.

\section{Conclusions}

We developed a high-performance pipeline for coherent dedispersion observations of the YNAO$40 \mathrm{M}$ telescope. The pipeline has been used in YNAO$40 \mathrm{M}$ for its routine observation everyday.

Overall, with DPDK and GPU techniques, our implementation of a coherent dedispersion algorithm on a multi- node, multi-device cluster allows data processing rates of $4 \mathrm{~Gb} / \mathrm{s}$ per node and $16 \mathrm{~Gb} / \mathrm{s}$ system-wide, which presents a valuable reference for other radio telescopes, especially for those based on hybrid FPGA/GPU architectures.

\section{Acknowledgements}

The work discussed in this paper was jointly supported by the Yunnan Astronomical Observatories of Chinese Academy of Sciences and the Kunming University of Science and Technology.

This work is supported by the National Key Research and Development Program of China (2018YFA0404603), the Joint Research Fund in Astronomy (No. U1831204, U1631129) under cooperative agreement between the National Natural Science Foundation of China (NSFC) and the Chinese Academy of Sciences (CAS), the Yunnan Key Research and Development Program (2018IA054), the Applied Basic Research Foundation of Yunnan Province (2017FB001), and the major scientific research project of Guangdong regular institutions of higher learning (2017KZDXM062).

This work is also supported by the Astronomical Big Data Joint Research Center, co-founded by National Astronomical Observatories, Chinese Academy of Sciences, and Alibaba Cloud. The authors also gratefully acknowledge the helpful comments and suggestions of the reviewers.

\section{References}

[1] Bassa C, Pleunis Z, Hessels J. Astronomy and Computing, 2017, 18(40).

[2] David H E M, Danny C P, Matthew L, et al. Publications of the Astronomical Society of the Pacific, 2018, 130:044502.

[3] DuPlain R, Ransom S, Demorest P, et al. in Advanced Software and Control for Astronomy II, Vol. 7019 (International Society for Optics and Photonics), 2008:70191D.

[4] Edwards R T, Hobbs G, Manchester R. Monthly Notices of the Royal Astronomical Society, 2006, 372:1549.

[5] Ford J M, Prestage R M, Bloss M. in Software and Cyber infrastructure for Astronomy III, Vol. 9152 (International Society for Optics and Photonics), 2014:915218. 
[6] Gallenmller S, Emmerich P, Wohlfart F, Raumer D, Carle G. in Proceedings of the Eleventh ACM/IEEE Symposium on Architectures for networking and communications systems (IEEE Computer Society), 2015:29-38.

[7] Hankins T. The Astrophysical Journal, 1971, 169:487.

[8] Hankins T H, Rickett B J. Meth. Comp. Phys, 2012,14 (55).

[9] Hargreaves J. in Millimeter, Sub millimeter, and Far-Infrared Detectors and Instrumentation for Astronomy VI, Vol. 8452 (International Society for Optics and Photonics), 2012:84522M

[10] Hickish J, Abdurashidova Z, Ali Z, et al. Journal of Astronomical Instrumentation, 2016, 5:1641001.

[11] Hotan A, Van Straten W, Manchester R. Publications of the Astronomical Society of Australia, 2004, 21:302.

[12] Karuppusamy R, Stappers B, Van Straten W. Publications of the Astronomical Society of the Pacific, 2008, 120:191.
[13] Kocz J, Greenhill L, Barsdell B, et al. Journal of Astronomical Instrumentation, 2015, 4:1550003.

[14] Lan C, Hao X, Chuang G, Mei Song T. in 2016 Progress in Electromagnetic Research Symposium (PIERS), 2016:2969-2972.

[15] Lorimer D R, Kramer M. Handbook of pulsar astronomy, Vol. 4 (Cambridge University Press), 2005.

[16] Manchester R, Taylor J. Astrophysical Letters, 1972, 10(67).

[17] Nie J, Pei X, Wang N, Chen M, Zhang H. Scientia Sinica Physica, Mechanica \& Astronomica, 2017, 47:059512.

[18] Recnik A, Bandura K, Denman N, et al. in Application-specific Systems, Architectures and Processors (ASAP), 2015 IEEE 26th International Conference on (IEEE), 2015:5761.

[19] Van Straten W, Bailes M. Publications of the Astronomical Society of Australia, 2011, 28(1). 